\title{
Learning The Fluid/Flow Properties Using Microfluidics
}

\author{
Pooria Hadikhani ${ }^{1, *}$, Navid Borhani $^{1}$, S. Mohammad H. Hashemi ${ }^{1,2}$, and Demetri Psaltis ${ }^{1}$ \\ ${ }^{1}$ Optics Laboratory, School of Engineering, Swiss Federal Institute of Technology Lausanne (EPFL), Lausanne, Switzerland \\ ${ }^{2}$ Computational Science and Engineering Laboratory, ETH Zurich, 8092 Zurich, Switzerland.
}

\begin{abstract}
Deep neural networks (DNN) are employed to measure the flow rate and the concentration of the liquid using the images of the droplets in a microfluidic device. The trained networks are able to measure flow rates and concentrations with good accuracy.
\end{abstract}

\section{Introduction}

Flow and fluid properties are crucial parameters that define the droplet flow properties in a microfluidic device. Due to the nonlinear dynamics of fluid flows, it is difficult to extract these properties using the governing equations of fluids. Neural networks can extract the fluid properties from images of their flow patterns since they can deal with nonlinear problems. The schematic of this procedure is shown in Fig. 1. Using the methodology of this study, the trained network is able to identify correctly flow rates and solution concentrations it has not seen before in the training set.

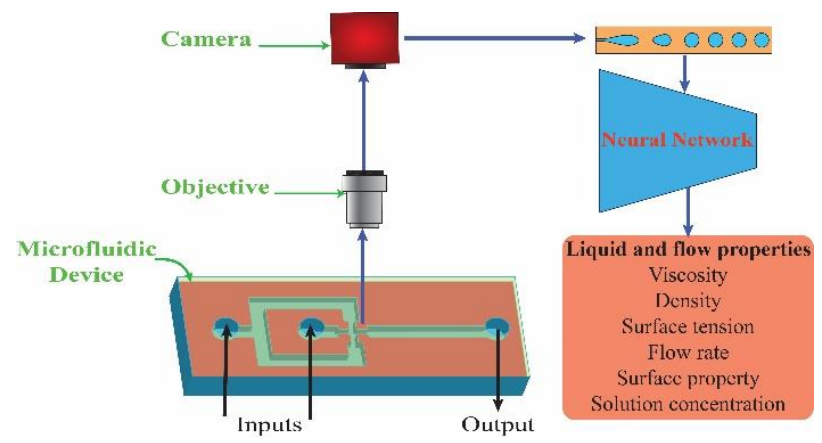

Fig. 1. Images of droplet formation in a microfluidic channel are used to train a neural network to extract information about the liquid and flow properties.

\section{Methods}

A flow focusing geometry is used to generate water-IPA droplets in silicone oil. The microfluidic device is fabricated in PDMS and bonded to glass. The flow rates are controlled using two syringe pumps. Images of droplet flows are captured using a microscope and a high-speed camera. For the flow rate measurement, the flow rate varies from $0.1 \mathrm{ml} / \mathrm{h}$ to $1.5 \mathrm{ml} / \mathrm{h}$ with $0.05 \mathrm{ml} / \mathrm{h}$ step. In this experiment the concentration of water-IPA is at constant concentration of $5.5 \%$. For the concentration measurement, the concentration of IPA in water is changing from $4 \%$ to $7 \%$ with $0.5 \%$ pitch. A regression neural network shown in Fig. 2 is implemented in Tensorflow library for the flow rate and concentration measurement. The network has three sets of convolutional/max pooling layer following by a fully connected layer. This network has a single output which gives the fluid property.

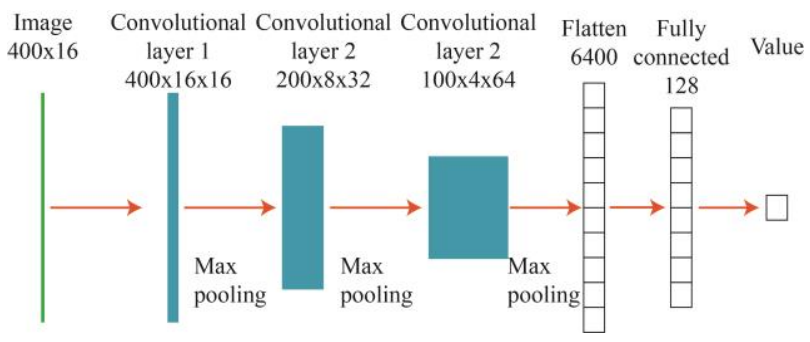

Fig. 2. Structure of neural network.

\section{Results}

Fig. 3a shows some instances of droplet flows at different flow rates. Images of flow rates from $0.1 \mathrm{ml} / \mathrm{h}$ to $1.5 \mathrm{ml} / \mathrm{h}$ with $0.1 \mathrm{ml} / \mathrm{h}$ step are used to train the network. Afterwards, new images of the same range of flow rate with $0.05 \mathrm{ml} / \mathrm{h}$ pitch are used for the test. Error of measured values by the DNN is shown in Fig. $3 b$. The mean error of the measurement for the trained and new flow rates are $2.878 \%$ and $5.729 \%$, respectively. The results show the capability of the DNN in interpolating between the trained values for the cases that is new for the network. For new flow rates, there are some cases that the measured value is different from the true value which is caused by sudden change of droplet flow regime.

* Corresponding author: pooria.hadikhani@epfl.ch 
a

\section{Flow rate $(\mathrm{ml} / \mathrm{h})$}

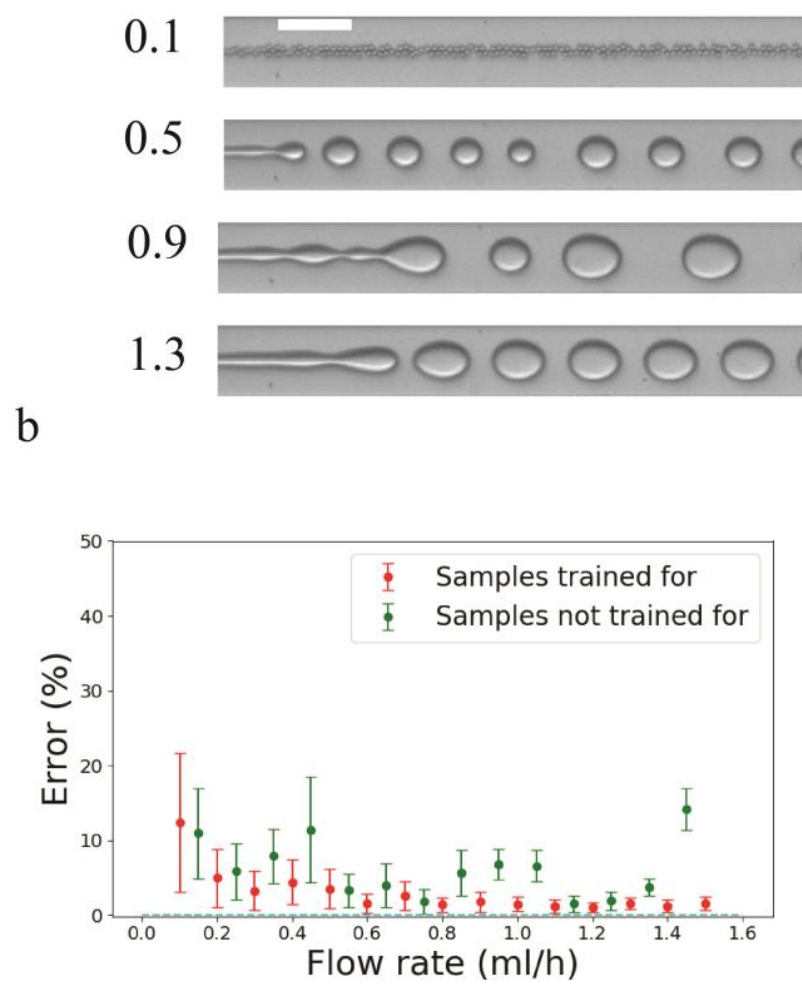

Fig. 3. Flow rate measurement: (a) Images of droplets at different flow rates. (b) Error of measured values by the network at different flow rates.

As can be seen in Fig. 4a, the concentration of isopropanol in the water is affecting the droplet flow pattern. The neural network is trained with samples ranging from $4.0 \%$ to $7.0 \%$ with $1.0 \%$ step. The network is tested with concentrations in the same range but with $0.5 \%$ steps. Due to a flow regime transition between concentrations of $5.0 \%$ and $6.0 \%$, the mean error of measurement for the new concentrations is $9.306 \%$. Because the flow regime transition is dependent on velocity as well as the concentration, we trained six networks at six different velocities to measure the concentration. Maximum three outliers of the measured values are removed and the closest measurement to the average is considered as the concentration. Furthermore, the output is calculated by averaging over the measurements for ten sample images input. This procedure decreases the mean error of measurement for the concentrations outside of the training set to $2.583 \%$. Fig. 4b shows the error of measurement at each concentration.

\section{Concentration}
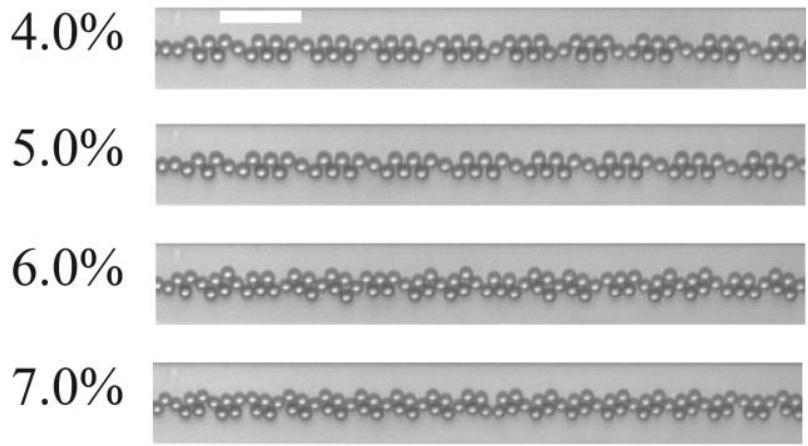

b

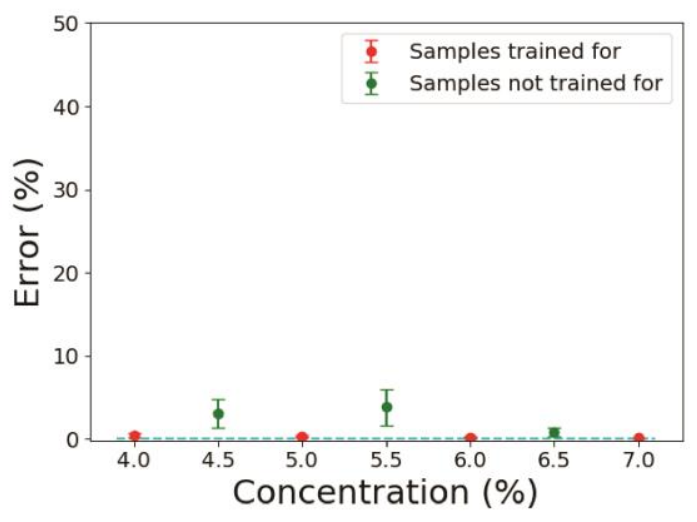

Fig. 4. Concentration measurement: (a) Images of droplets at different concentrations. (b) Error of measured values by the network at different concentrations.

\section{Conclusion}

In this study, we used deep neural networks to extract information about the flow/liquid properties from droplet flow images. As an example, DNNs are used to measure the flow rate of the flow and the concentration of the liquid components. DNNs can be used to discover other fluid/flow properties such as viscosity, surface tension, material surface property, and density. This method has potential to be extended to measure multiple fluid properties from a single image window on a microfluidic chip. 